\title{
Outside-the-(Cavity-prep)-Box Thinking
}

\section{V.P. Thompson ${ }^{1 *}$, T.F. Watson', G.W. Marshall, Jr. ${ }^{2}$, B.R.K. Blackman ${ }^{3}$, J.W. Stansbury ${ }^{4}$, L.S. Schadler ${ }^{5}$, R.A. Pearson ${ }^{6}$, and R. Libanori ${ }^{7}$}

${ }^{1}$ Biomaterials, Biomimetics and Biophotonics Group, King's College London Dental Institute, London, UK; ${ }^{2}$ Preventive and Restorative Dental Sciences, University of California San Francisco, San Francisco, CA, USA; ${ }^{3}$ Department of Mechanical Engineering, Imperial College London, South Kensington Campus, London, UK; ${ }^{4}$ University of Colorado School of Dental Medicine, Boulder, CO, USA; ${ }^{5}$ Department of Chemical Engineering, Rensselaer Polytechnic Institute, Troy, NY, USA; ${ }^{6}$ Materials Science and Engineering, Lehigh University, Bethlehem, PA, USA; and ${ }^{7}$ Complex Materials, Department of Materials, ETH Zürich, Switzerland; *corresponding author, van.thompson@kcl.ac.uk

Adv Dent Res 25(1):24-32, 2013

This is information presented at the IADR Dental Materials Innovation Workshop, December 10-12, 2012, King's College London, UK. Sponsored by the International Association for Dental Research, FDI World Dental Federation, World Health Organization, United Nations Environmental Programme, and King's College London Dental Institute.

\section{ABSTRACT}

Direct placement restorative materials must interface with tooth structures that are often compromised by caries or trauma. The material must seal the interface while providing sufficient strength and wear resistance to assure function of the tooth for, ideally, the lifetime of the patient. Needed are direct restorative materials that are less technique-sensitive than current resinbased composite systems while having improved properties. The ideal material could be successfully used in areas of the world with limited infrastructure. Advances in our understanding of the interface between the restoration adhesive system and the stages of carious dentin can be used to promote remineralization. Application of fracture mechanics to adhesion at the tooth-restoration interface can provide insights for improvement. Research in polymer systems suggests alternatives to current composite resin matrix systems to overcome technique sensitivity, while advances in nano- and mesoparticle reinforcement and alignment in composite systems can increase material strength, toughness, and wear resistance, foreshadowing dental application.

\section{DOI: $10.1177 / 0022034513502207$}

(C) International \& American Associations for Dental Research
$\mathbf{T}$ he patency of the tooth-restoration interface is critical to the restored tooth's vitality, function, and appearance. The interfacial position within the damaged tooth and the physical quality of the materials involved in this complex have profound effects upon the likely success or failure of the restoration. The challenges presented to operative dentists and materials scientists arise from the need for handling the tissues in a biologically sound manner and then using materials intelligently to achieve optimal results. The need for this intervention is from the major cause of tooth tissue loss - dental caries - but the problems of an aging population with increasing levels of tooth wear also present challenges in both operative techniques and materials application. Clearly, the patient (and government, if funding dental care) would like all techniques to be faster, better, and cheaper - goals to which dental professionals may also aspire.

Restorations involve a series of linked and interplaying dental structures between the depths of the tooth cavity and the restorative material. Failure can occur from weak links between the tooth enamel or dentin and adhesive, the adhesive itself, the adhesive/ restoration boundary, and, finally, the restoration itself. Most materials research uses dental substrates and materials handling techniques that are operating under close-to-perfect conditions and bear little reality to the difficulties that may be encountered clinically. The tooth-restoration interface is problematic, given that we normally start with damaged tissue, whether that is due to the disease process or to our operative management.

When we prepare tooth tissue with a bur, we actually fracture the enamel rather than cut it, leaving behind residual cracks and fault lines within the prismatic structure (Watson et al., 2008). These fault lines can easily open and lead to white lines at cavity margins, due to shrinkage of a bonded restorative material bending and pulling apart the enamel prisms in their relatively weak lateral axis. Particle abrasion preparation techniques with powders of variable hardness, such as bioactive glass, may be selective for removing only weakened enamel and will produce much less subsurface enamel damage (Banerjee et al., 2011). The smeared surface produced by many dental operative interventions, including burs, is considered poor for adhesion and is often contaminated with mixed materials, e.g., soft tissues, bacteria, and degraded hard-tissue pressure welded into the surface by the "cutting" process.

Whether to remove all carious tissue is controversial, but there are numerous studies that show sealing of caries to have a

\section{Key Words}

nanocomposites, cross-link density, remineralization, biomimetics, dentin bonding, aligned composites. 
beneficial effect, e.g., the original ten-year study from MertzFairhurst and co-workers (1998) and the study by Paddick et al. (2005). These studies rely on the presence of an effective marginal seal, usually dependent upon an intact enamel margin. The concept of zones of infected and affected carious dentin is oversimplified in terms of the true mixing of the bacterial penetration and their products within the porous dentin structure, as well as the physico-chemical and pathological healing response of the dental pulp. The physical properties of the dentin will be weakest at the lesion surface, where the collagen is denatured, the bacterial load is high, and the field very wet. This surface creates the greatest challenge for achieving a satisfactory link between restoration and tooth, whether mediated by an adhesive or infiltrated by restorative material components. Not surprisingly, self-limiting caries excavation techniques have developed to remove weak material, e.g., chemo-mechanical (Carisolv), plastic burs, and air carrier particle abrasion, to leave a reasonably solid foundation and reduce the risk of pulpal exposure. Modern materials can be engineered to interact favorably with these prepared surfaces (Banerjee et al., 2010).

Modern dentin bonding systems work well in the 'perfect' environment of the testing laboratory with sound teeth as a substrate. Even so, there is a consistent problem of breakdown of adhesive bonds when samples are stored in water over a few months. This is mainly due to degradation of exposed collagen from the release of endogenous matrix metalloproteinases (MMPs) and other protease enzymes. Various strategies have been applied to reduce this effect, such as the use of chlorhexidine on the cavity surface and MMP inhibitors incorporated into the adhesive components (De Munck et al., 2009; Almahdy et al., 2012). As with many modifications to adhesives and materials, the clinical benefits of such strategies will take time to become apparent.

An alternative approach to tooth interface management is re-mineralization by biomimetic techniques or materials that simply encourage mineralization of the interface. Many of these materials are water-based cements. It is well-recognized that glass-ionomer cements (GICs) can undergo an ionic exchange with tooth tissue (Lin et al., 1992). The Atraumatic Restorative Treatment (ART) technique was developed with conventional GICs as the restorative material most capable of being used in adverse operating conditions. Such materials can be used as transitional (stabilizing) restorations for the management of a patient with extensive caries. The removal of the reservoir of bacteria from badly damaged teeth and the provision of a cleansable tooth surface are of great benefit in the overall management of such cases.

The new calcium tri-silicate cements also show promise as materials capable of inducing mineralization of dentin, with a mechanism of interaction based upon the alkalinity of the cement changing the collagen adjacent to the cement and developing a mineral interaction zone in this region (Atmeh et al., 2012). Such mechanisms could be of benefit in reinforcing the carious dentin interface, while alkaline materials will also have a pulpal therapeutic effect and be bio-inductive.

There is a need for operative techniques that preserve and conserve the dental hard tissues as much as possible. Materials should be functionally sound and simple to use, with serious consideration being given to improve the bond stability of the tooth-restoration interface by using biomimetic and bio-inductive materials.

\section{REMINERALIZATION-PROMOTING DENTAL MATERIALS}

Modern invasive dentistry seeks to preserve and restore native tissues to as great an extent as possible, e.g., enamel remineralization via fluoride treatments and, more recently, an amelogeninassisted approach (Fan et al., 2012). Dentin remineralization is more challenging, because dentin is more complex than enamel, being an inherently moist biological composite and, like bone, consisting mainly of collagen type I reinforced with apatite at the nanoscale.

Clinical observations suggest that caries lesions may arrest and reverse under the right circumstances. As noted above, clinical trials of the ART method show that cavity sealing with GICs may promote such arrest and reversal. Enamel remineralization treatments are available, and new approaches based on calcium-, phosphate-, and fluoride-releasing nanocomposites provide significant added promise. A recent review is available (Xu et al., 2010), and nanoACP-containing resin-based composites have shown evidence of significant caries inhibition and enamel remineralization (Weir et al., 2012; Melo et al., 2013). Dentin remineralization, however, is in its early stages. Most studies have focused on delivering calcium and phosphate ions to promote regrowth of apatite via classic nucleation and growth from solution. Emerging is an understanding that amorphous calcium phosphate $(\mathrm{ACP})$ plays an important role in biomineralization.

\section{Calcium and Phosphate Solutions and Materials for Remineralization}

ten Cate (2001) evaluated remineralization of artificial caries lesions in enamel and extending $200 \mu \mathrm{m}$ into dentin. The artificial lesions were treated with several fluoride variations, an inhibitor, and calcium phosphate solution controls. Experimental treatments caused mainly outer enamel remineralization and were best with fluoride. Interestingly, the control calciumphosphate solution was best in the deepest part of the dentin lesion. This suggests that significant dentin remineralization occurred beneath the enamel lesion from the depth toward the surface, although the process was slow.

Peters et al. (2010) conducted a randomized clinical trial of remineralization via a resin-based $\mathrm{Ca}-\mathrm{P}$ cement used as a base under composite as compared with no-base controls. Carious and sound teeth scheduled for extraction were prepared by conservative cavity preparation including the use of self-limiting polymer burs. Clinical evaluation showed re-hardening after 3 months in vivo, and later electron microprobe study indicated restoration of normal calcium and phosphate levels.

Mineral trioxide aggregate (MTA) has been widely studied for pulp capping and root retrofilling. A commercial calcium silicate material (Biodentine), suggested for replacement of coronal dentin, releases $\mathrm{Ca}, \mathrm{OH}$, and $\mathrm{CO}_{3}$ ions on hydration. Atmeh et al. (2012) compared it and GIC interactions with dentin on hydration in the absence of a phosphate ion source. Biodentine resulted in a mineral infiltration zone that penetrated 
the tubules, reminiscent of MTA, but also infiltrated the intertubular dentin. Infiltration was facilitated by $\mathrm{CaOH}$ caustic etching of the collagen matrix, leaving the peritubular dentin intact. GIC penetrated dentin through a distinctly different acidic mechanism without significantly altering the collagen.

Several studies have evaluated a variety of $\mathrm{Ca}-\mathrm{P}$ solutions in the remineralization of artificial caries lesions (Bertassoni et al., 2010, 2011). Simple storage in solutions often produced surface precipitation yielding limited recovery of properties. In contrast, a constant composition approach (Bertassoni et al., 2011) did not yield surface precipitates, chemical and mechanical property recovery was greater, and remineralization occurred from the depth of the lesion toward the surface. These and other studies indicate that dentin remineralization to restore properties depends on regrowth of remnant crystals within the intrafibrillar zone of collagen fibrils (Kinney et al., 2001, 2003).

\section{Biomineralization and Amorphous Calcium Phosphate Precursors}

A second route to remineralization exists based upon biomineralization mediated by amorphous $\mathrm{Ca}-\mathrm{P}$ precursors (ACP) and polyanionic polymers. Many biominerals appear to be produced following formation of an ACP phase rather than the classic nucleation and growth from solution (Gower, 2008). Biominerals contain large quantities of soluble acidic proteins thought responsible for specific interactions to nucleate and control crystal morphology. However, Gower (2008) has suggested that they serve as "process directing agents", and her group developed a system based on polyaspartic acid (polyAsp) that has produced interesting structures for both calcium carbonate and for $\mathrm{Ca}-\mathrm{P}$ collagen matrices. Collagen by itself does not readily promote epitaxial growth within the fibrils from saturated $\mathrm{Ca}-\mathrm{P}$ solutions. Gower further suggests that the ACP precursor may be fluidic and may involve a polymer-induced liquid-precursor (PILP) process based on polyAsp to mimic many of the features seen in biomineralization. The PILP process allows for intrafibrillar mineralization in a variety of collagen I matrices, as well as demineralized dentin, and provides apatite crystals that align as in normal bone and dentin. Nudelman et al. (2010) showed ACP precursors/nanodroplets induced by polyAsp entering the collagen fibrils at the gap-overlap region, areas of high positive charge. They concluded that type I collagen aided by such droplets can nucleate intrafibrillar mineralization.

\section{Dentin Functional and Biomimetic Remineralization}

For reconstruction of dentin, re-establishing the intrafibrillar apatite mineral is critical (Bertassoni et al., 2009). In 2008, Tay and Pashley began publishing studies on biomimetic remineralization aimed at protective remineralization of dentin hybrid layers (surface-demineralized zones) to stabilize them and prolong clinical lifetimes. They used polyacrylic acid (PAA) as a fluidic precursor and a second polyanionic acid, PVPA, thought necessary as a template for mineral within the collagen fibrils. This system aids in mineralization of acid-demineralized collagen fibrils and completely demineralized dentin matrices (Liu et al., 2011). These biomimetic analogs can be included in MTA for remineralization of artificial caries-like lesions (Qi et al., 2012).
Burwell et al. (2012) applied the PILP method to artificial caries lesions to promote functional remineralization, i.e., the recovery of the structure and properties of the dentin. Lesions $140 \mu \mathrm{m}$ deep were remineralized for 7, 14, and 28 days and evaluated for mineral and mechanical property recovery. Increased lesion remineralization occurred after 7, 14, and 28 days (Fig. 1a). Mineral recovery proceeded from the depth to the surface and was nearly complete at 14 days (Fig. 1b). Increases in elastic modulus (Fig. 1c) showed that the sloped part regains mechanical properties quickly. The highly demineralized flat zone regained mineral more slowly, and although full mineralization was regained throughout the lesion, the flat zone gained about $50 \%$ to $60 \%$ of normal modulus values. TEM/electron diffraction showed intrafibrillar mineral formation with crystal size and orientation increasing with time and similar to normal dentin.

\section{Summary}

Re-establishing intrafibrillar mineral levels is critical for the recovery of dentin structure and properties. PILP and related methods can provide substantial functional remineralization in artificial caries. New evidence shows that polyanionic polymers induce intrafibrillar mineralization, and this may extend to softer carious regions that are normally removed during conservative cavity preparations. Additional modifiers may promote further structural and property recovery. Calcium and phosphate ions seem to be effective in recovery where remaining mineral is present.

\section{ADHESION AND FRACTURE MECHANICS}

As noted above, the bonding of restorations to teeth clearly presents a significant challenge. Not only is the service environment hostile, but also the joint loadings can be significant, and the joints must be made in a very short time with restricted cure possibilities and significant toxicity constraints. In the field of structural bonding, it is common to have one or two of these issues occurring simultaneously but rarely do they all occur together. Various structural bonding problems may be considered, and the strategies developed for their solution may be analyzed for potential benefits by the use of common approaches.

Fracture mechanics approaches have been key to achieving success in structural bonding. A relevant example is the joining of titanium alloy protection strips to carbon-fiber-reinforced composite fan blades in an aircraft turbo-fan engine. Here, materials with very different elastic properties are fixed in a joint that experiences elevated temperatures (around $90^{\circ} \mathrm{C}$ in flight), frequent exposure to water and other chemicals, and complex loading due to centripetal forces, vibration deformation modes, and aerodynamically induced stresses. Critical issues are the selection of effective surface pre-treatments for the dissimilar substrates and the long-term durability of the epoxy adhesive bond, with a service life of 20 years being required. Fracture mechanics test specimens provide basic fracture resistance values for the joints in the various modes of loading, and these can also be used to measure the resistance of the joint to repeated loading (fatigue) in a hostile environment (e.g., water) to gauge the long-term reliability. Fracture mechanics data are combined with analytical and/or with numerical FEA models of the joint to predict the lifetime of the joint under known loading 
conditions and defined environments. Biologic variation in the composition and geometry of teeth confounds such studies.

Another relevant example is the joining of aluminum extrusions in a high-performance sports car. Here, similar materials were bonded, and a key requirement of the joint was to ensure that a high-energy deformation mode of failure occurred in the event of a vehicle collision. Thus, sufficient interfacial adhesion and adhesive toughness are required to trigger the large-scale plastic deformation of the metallic extrusions and hence dissipate the kinetic energy of the vehicle in the event of a crash. A critical issue here is the rate sensitivity of the epoxy paste adhesive, since the loading duration is a few milliseconds during a crash. Thus, a characterization of the fracture behavior at quasi-static test rates (failure duration of several minutes) was not appropriate, and high-speed fracture mechanics testing, analysis, and modeling were required (Blackman et al., 2009). It is appropriate to note that mastication loading of teeth occurs over 10 to 50 milliseconds.

The final 'structural' bonding example is bonding between the aggregate (the substrate) and bitumen (the adhesive) in asphalt mixtures, as commonly used as road surface materials. The poor state of many road surfaces is testament to the failure of the aggregate stone-to-bitumen weak adhesive interface, which opens paths for moisture ingression which then is accelerated with winter freeze-thaw crack cycling. This adhesion problem required the development of a peel test specimen such that the interface between bitumen and selected aggregate materials could be directly investigated. In addition, strategies for the moisture exposure of the joint had to be developed and a fracture mechanics protocol for this unique application developed (Blackman et al., 2013). Such techniques must yield results independent of test specimen geometry, a key feature of the fracture mechanics approach. With this tool, the individual durability of various combinations of aggregate and bitumen was assessed, and problem combinations were identified. Efforts to improve the interfacial adhesion between any given aggregatebitumen combination are being pursued.

As highlighted in the examples above, a fracture mechanics approach, incorporating the loading mode and the service environment, is an important technique. However, to translate the approach to dental bonding and to provide answers to key questions requires both the development of new experimental tests and the formation of suitable and validated models to describe the performance of the bond. Experimentally, the small size of the required test specimens poses significant challenges, since the requirement for constraint is usually achieved (for example, in aerospace bonding) by the use of sufficiently large test specimens. However, the need to minimize specimen size is currently important in several fields, e.g., in the bonding of electronic components, so some cross-over of techniques between these applications is certainly possible. Turning to the models, then, accurate input parameters are the key to obtaining useful results. Requirements include the elastic response of the joint material, adhesive fracture energy, and a realistic description of the damage processes occurring as the joint degrades from its initial state to its fully damaged (broken) state. These damage formulations can be implemented in fatigue (to predict allowable loading levels for a target life) and to correctly take loading rate and

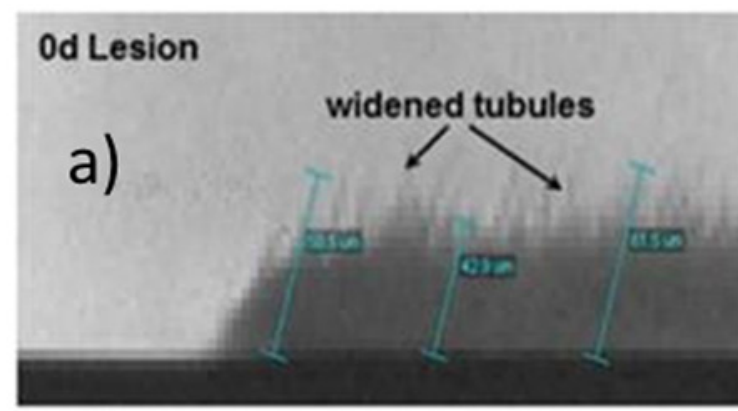

\section{4d Remin.}
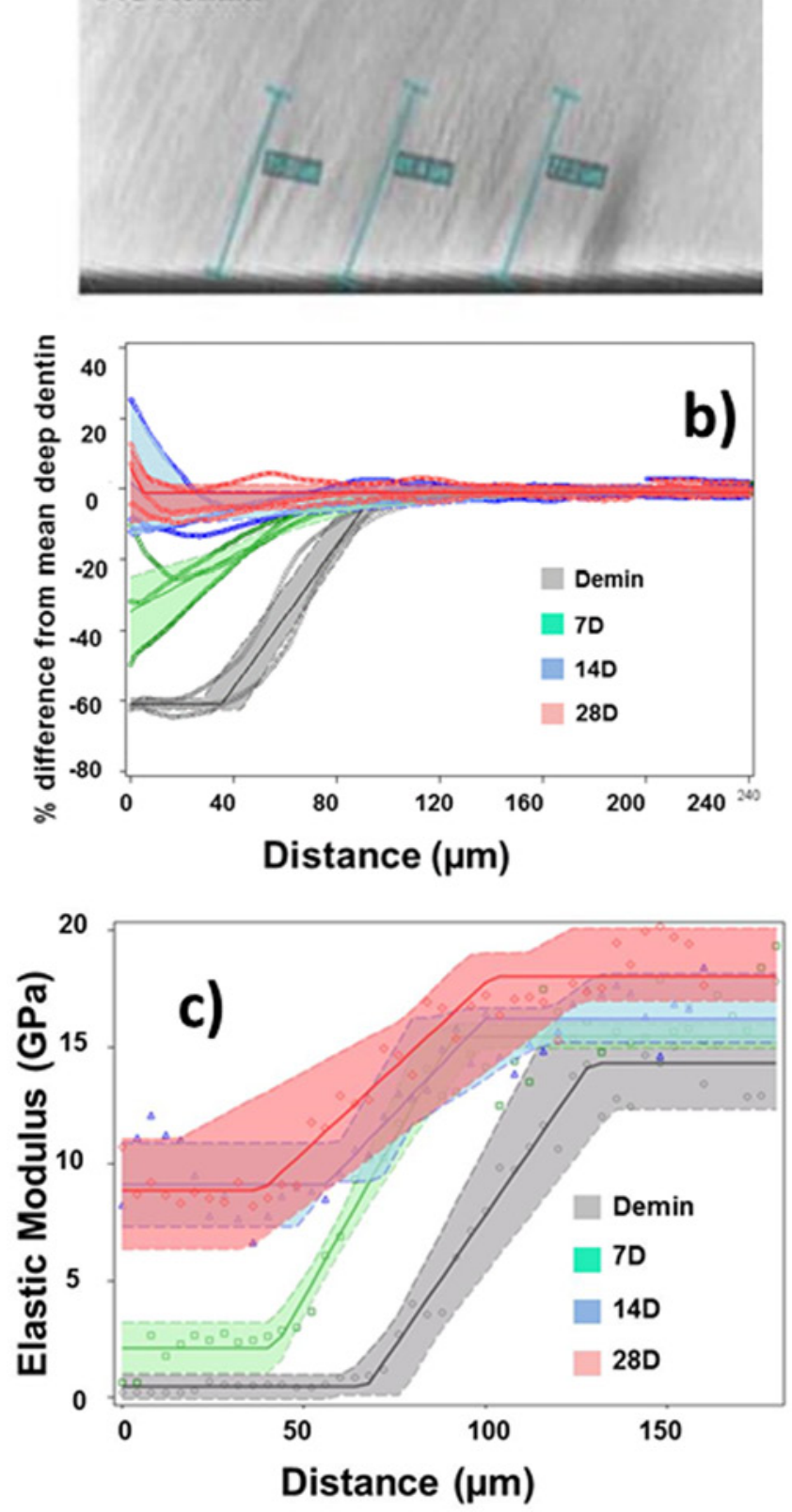

Figure 1. PILP remineralization of artificial caries. (a) MicroXCT images of lesion and 14-day treatment. (b) Mineral profiles of lesion, 7-, 14and 28-day treatments. (c) Reduced elastic modulus changes during mineralization from 0-, 7-, 14-, and 28-day treatments (nanoindentationderived). Modifed from Burwell et al. (2012). 
environmental effects into account. As an example, mastication loading rates are in the range of $1,000 \mathrm{~N} / \mathrm{sec}$, but most dental adhesive testing is conducted at 1 to $5 \mathrm{~N} / \mathrm{sec}$. Such validated models could then be used to assist in the optimization of joint design.

\section{ADVANCES IN POLYMER CHEMISTRY AND COMPOSITE RESIN SYSTEMS}

Polymer-based direct placement materials have become the most widely used direct dental restorative treatment option but are limited by a high degree of process-related detail in terms of tooth preparation, the application of the adhesive, and the photocuring of the composite required for success. This technique sensitivity relates to clinical performance and longevity concerns, including marginal gap formation, tooth sensitivity, development of adjacent secondary caries, and restoration fracture. Dimethacrylate monomers continue to dominate the dental resin formulations, with Bowen's BisGMA still one of the most commonly encountered components. However, given concerns related to bisphenol A (BPA) exposure and the fact that most BisGMA-based resins do not achieve high levels of conversion (from relatively high monomeric glass transition temperatures of approximately $-50^{\circ} \mathrm{C}$, which limits monomer mobility), there are wide-ranging efforts to design new resin systems that meet the demanding performance requirements and challenges imposed by in situ curing. The following are just some of the design criteria considerations for new resins: biocompatible components; a readily adaptable liquid matrix compatible with a prepared tooth interface and a well-dispersed, high filler loading; a reasonable refractive index match between filler and matrix; stability until activation by photopolymerization or other mode that then precipitates a fast, extended depth and high conversion reaction with well-controlled exothermic response; and a highly crosslinked or otherwise chemically resistant polymer that displays immediate high strength, modulus, and fracture toughness achieved with low shrinkage and stress development. A look at new directions in applicable areas of polymer chemistry and some examples of potential approaches are described here.

In the effort to replace BPA-based monomers in dental materials, which include BisGMA and the ethoxylated version (BisEMA) as well as urethane-modified BisGMA and related derivatives, several of the above issues complicate the simple substitution by other widely available methacrylate crosslinkers. One interesting BisGMA option is a urethane dimethacrylate (UDMA) and methacrylic acid (MAA) copolymer (Tanaka et al., 2001). With a stoichiometric balance between the acidic and urethane functional groups, the very modest-viscosity UDMA/ MAA resin converts to a remarkably strong and tough polymer network (flexural strength $\geq 250 \mathrm{MPa}$, even in the presence of water as compared with BisGMA-based polymers that are limited to $\sim 160 \mathrm{MPa})$. This comonomer composition shows the potential of designing specific secondary interactions into polymers to achieve excellent strength while enhancing toughness. The issue of refractive index matching can be addressed by the introduction of relatively low concentrations of high refrac- tive index prepolymerized nanoparticles. Nanogels can be introduced that, upon polymerization, co-react with the resin and contribute to polymer network structure while mitigating stress development (Moraese et al., 2011; Liu et al., 2012).

Currently there are incomplete, vitrification-restricted levels of conversion with most dimethacrylate resin formulations. High filler loading further compromises the conversion, with values of $50 \%$ to $60 \%$ not unusual among commercial dental composites. At these conversion levels, a significant amount $(10 \%$ to $20 \%$ ) of free monomer remains unreacted and potentially leachable (Stansbury and Dickens, 2001). The potential deterioration of mechanical properties over time as leached monomer is replaced by water is compounded by concern over local and systemic biocompatibility as well as alteration of oral biofilm composition and activity. Very high conversions in conventional dimethacrylate resins result in high internal stress polymers with no increase in fracture toughness. An intermediate approach to lessen monomer release involves use of bulky dimer acidderived dimethacrylates photo-copolymerized with conventional dimethacrylate comonomers with up to $83 \%$ conversion and equivalent strength to BisGMA/TEGDMA polymers at $64 \%$ conversion ( $\mathrm{Lu}$ et al., 2010). At this higher level conversion, approximately $95 \%$ of all monomer is covalently incorporated into the polymeric network. A different approach can be envisioned based on a two-stage polymerization reaction process where, in the first step, all free monomer is consumed to create a loosely crosslinked low-glass-transition-temperature polymer. A second-stage cure follows in which orthogonally reactive groups on the initial monomers combine to achieve the required highly crosslinked network structure. The vitrification limit of the secondary conversion leaves behind no leachable free monomer. Several approaches could deliver this result from a single photopolymerization exposure, as generally demonstrated with methacrylate and vinyl ether comonomers (Lin and Stansbury, 2003). Here, hybrid monomers containing both reactive functional groups would be necessary to achieve the single polymerization, which also would be expected to offer beneficially low polymerization stress.

There is exciting ongoing and upcoming innovation in the area of polymer chemistry directed toward improved dental restoratives. This is tempered by the very challenging constraints imposed by dental applications as well as by the regulatory issues that become more complex with greater departures from the status quo.

\section{POLYMER NANOCOMPOSITES}

Polymer nanocomposites have the potential for use as dental materials and can be defined as polymers containing fillers (spheres, plates, fibers) with one dimension smaller than 100 $\mathrm{nm}$. Fumed silica (400 $\mathrm{nm}$ in size) has been used to provide thixotropic behavior to dental composite resins for many years, while a version with agglomerated micron-sized particles, while of limited fracture toughness, has been available for over 20 years (Heliomolar, Ivoclar-Vivadent, Schaan, Liechtenstein) and used successfully in posterior restorations (Da Rosa Rodolpho et al., 2011). Given our new understanding of nanocomposites, 
they are of significant interest to dentistry for 3 reasons. First, the fillers often have properties that are different from the bulk properties of the same material. For example, barium titanate exhibits a marked change in dielectric constant with decreasing particle size (Wada et al., 2008). Second, well-dispersed nanoscale fillers are small defects. They are much smaller than the critical crack size that can lead to failure in a composite material and are often small enough that they do not significantly scatter light. Third, because of the large surface area of the fillers, nanocomposites have a large volume of interfacial matrix material (interphase) with properties different from those of the bulk polymer. These 3 attributes of nanocomposites lead to unique combinations of properties, not achievable in other composites, for example, composites with enhanced strength and ductility (Irwin et al., 2003; Ash et al., 2004) or scratchresistant, transparent coatings (Barna et al., 2005). The nanofillers can also assemble into mesoscale structures (Sides et al., 2006; Akcora et al., 2009) that are used as is or are heat-treated to create mesoporous ceramic structures (Stefik et al., 2012).

For use as dental materials, nanocomposites offer some interesting possibilities:

- It has been widely established that cell and protein interaction with a surface can be altered with nanostructured composites. For example, human mesenchymal cell proliferation increased significantly for surfaces with polyglycolic-lactic acid and nanohydroxyapatite used as scaffolds (Ravichandran et al., 2012). This could lead to better osseointegration (Tran et al., 2009).

- Nanoscale fibers can be produced in bulk, and oriented to create 3D structures with anisotropic properties to match those of the biological materials they replace, by techniques such as electrospinning (Akcora et al., 2009).

- 'Direct-write' manufacturing opens up the possibility of manufacturing implants on site that are exact matches to the bone being removed (Lewis et al., 2006).

- The large interfacial area between the nanofiller and matrix opens up the potential for tailoring mechanical properties to increase toughness and wear resistance (Gao et al., 2012).

In this option, $\mathrm{SiO}_{2}$ nanoparticles with grafted block copolymer consisting of a rubbery inner block and a matrix-compatible outer block were synthesized to toughen an epoxy matrix (Gao et al., 2012). The rubbery inner block was poly(hexyl methacrylate) (PHMA), and the epoxy-compatible outer block was poly(glycidyl methacrylate) (PGMA). A systematic study of the effect of block copolymer graft density and block molecular weight on the tensile, fracture toughness, and fatigue properties found that the copolymer-grafted $\mathrm{SiO}_{2}$ nanoparticles enhanced the ductility (maximum 60\% improvement), fracture toughness (Fig. 2; maximum $300 \%$ improvement), and fatigue crack growth resistance of the epoxy matrix while maintaining the modulus, with less than 2 vol\% $\mathrm{SiO}_{2}$. Plastic void growth and shear banding were found to be the main toughening mechanisms.

The challenges for the use of nanocomposites are primarily in 3 areas: (1) The increased viscosity due to nanoparticles can limit nanoparticle loading, (2) the nanoparticle dispersion can be

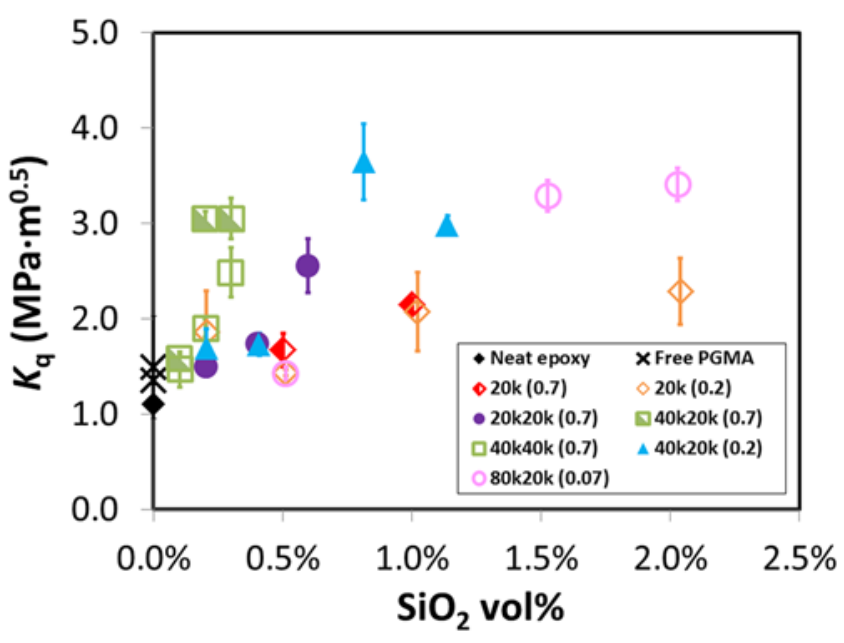

Figure 2. The fracture toughness of diblock copolymer-modified silica nanoparticles in an epoxy matrix as a function of loading. The legend shows each sample made. The first number corresponds to the molecular weight of the inner rubbery block, while the second number is the molecular weight of the PGMA-compatible layer. The number in parentheses is the graft density in chains $/ \mathrm{nm}^{2}$ of the grafted polymer. When there is only one number, it corresponds to a filler modified with PGMA only.

difficult to control during commercial processing, and (3) the environmental and health impacts of some nanoparticles are unclear. For modification of polymers for dental materials, all 3 of these challenges can be overcome, and this is clear from the use of nanocomposites in many commercial fields (rubber tires, sunscreen, etc.).

In summary, the latest polymer nanocomposites suggest the potential for the creation of tougher and harder dental materials with improved wear resistance that can be tailored for direct restorations. The potential also exists for self-healing dental materials with bacterial resistance.

\section{NANOMATERIALS AND INCREASING TOUGHNESS}

Epoxy resins are inherently brittle because of their crosslinked nature and are not unlike current dental BisGMA composite matrix resins in this regard. Fortunately, there are several additive approaches that have been shown to increase the fracture resistance of epoxies which may be applicable to dental resin systems. Traditionally, such additives were micron-size in dimensions, but recently a plethora of nanoscale alternatives has been developed. A side-by-side comparison of the toughness enhancement with both micron- and nanometer-size additives in a model epoxy allows for an understanding of the toughening mechanisms.

Several investigators have shown micron-size glass spheres to be effective toughening agents for a BisA epoxy cured with piperidine. Kawaguchi and Pearson (2003) have shown that glass spheres with a mean diameter of 42 microns can increase the fracture toughness of this model epoxy resin by over $50 \%$. Microscopy techniques revealed that the toughening mechanisms involved crack-tip-pinning as well as particle bridging in 

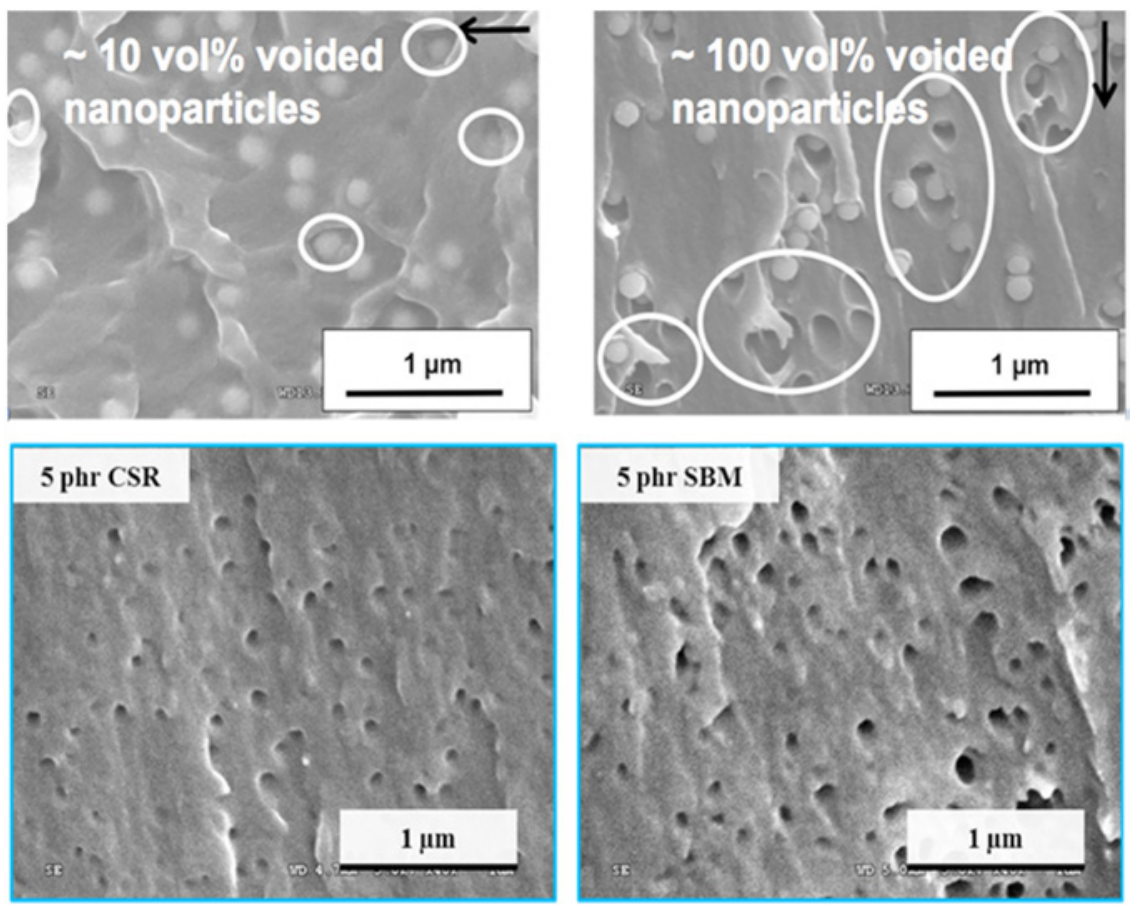

Figure 3. Scanning electron micrographs reveal that hard silica nanoparticles debond from the epoxy matrix (top), whereas soft rubbery nanoparticles cavitate (bottom). Arrows indicate crack growth direction, CSR = core shell rubber particles, SBM = poly/styrene-block-butadieneblock-methyl methacrylate) particles. phr = parts per 100 resin (which is the amount of filler/ modifier per 100 grams of epoxy resin).

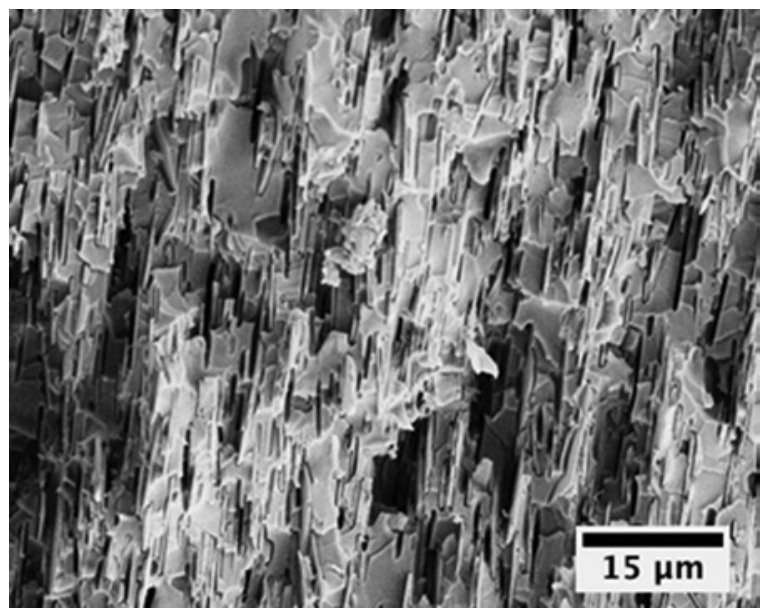

Figure 4. Fractured surface of a bio-inspired platelet-reinforced composite obtained by magnetic manipulation of the modified alumina platelets (volume fraction $=0.25$ ).

the crack wake. The increases in toughness agree well with predictions from use of the crack-tip-pinning and bridging model (Rose, 1987). Increases in modulus and strength were also observed and can by predicted by traditional micromechanical models.

Nanometer-size silica particles, ranging from $23 \mathrm{~nm}$ to 170 $\mathrm{nm}$, have been shown to be more effective toughening agents for the same model epoxy with fracture toughness increases of over $100 \%$. Electron (Fig. 3, upper images) and optical microscopy revealed that the toughening mechanisms were fundamentally different from those of micron-size filled epoxies and involved nanoparticle-induced shearbanding and matrix void growth (Dittanet and Pearson, 2012). The increases in toughness agree well with predictions from the model used by Johnsen et al. (2007), when one considers that only $10 \%$ of the nanosilica particles debond. Interestingly, the addition of micron-size particles can induce nearly $100 \%$ of the silica nanoparticles to debond (see upper right micrograph in Fig. 1) (Dittanet and Pearson, 2013).

Some epoxy resins can readily shear yield, which provides the ability to increase toughness by a particle additive approach. Specifically, reducing crosslink density of the epoxy matrix increases the "toughenability" for both rubber-toughened (Pearson and Yee, 1989) and filled epoxies (Lee and Yee, 2000). However, rubber modification of epoxies has been shown to be the more effective approach for lightly crosslinked resins.

There is also a particle size effect for rubber-toughened epoxies. Fracture toughness increases of over $400 \%$ have been observed (Pearson and Bacigalupo, 2011)] in the same model epoxy system mentioned above. Several types of particles were examined: $\sim 3$-micron-diameter CTBN-particles $(\mathrm{CTBN}=$ carboxyl terminated, random copolymers of butadiene and acrylonitrile), 50-nm-diameter CSR (CSR = core-shell rubber particles), and $\sim 40$-nm-diameter SBM [poly(styrene-block-butadiene-block-methyl methacrylate)]. The toughening mechanisms were qualitatively similar for all 3 types of rubber particles and involved massive shear banding and matrix void growth at the crack tip (Fig. 3, lower images). The advantage of the self-assembling SBM block copolymers is that toughness improvements were attained with one-third the amount of rubber, so decreases in modulus and strength were minimal.

In summary, recent advances in nanoscale toughening agents for epoxy resins provide significant improvements in toughness. Such advances are already being applied to vinyl ester resins and should be readily transferable to dental-resin-based materials.

\section{BIO-INSPIRED COMPLEX MATERIALS}

Although composite materials provide improved esthetics in dental restorations, they still exhibit several disadvantages as compared with the conventional amalgam, such as low hardness and toughness and limited resistance against wear and impact 
(Cramer et al., 2011). In contrast, biological composites perform extremely well when loaded in any given direction, even though they are comprised of relatively weak building blocks, such as hydroxyapatite and calcium carbonate (Jackson et al., 1988; Studart, 2012). Combining the wealth of chemical compositions available in synthetic materials with the microstructural design principles naturally used in biological systems allows for the design and fabrication of bio-inspired composites that exhibit unusual combinations of mechanical properties (Tang et al., 2003; Bonderer et al., 2008; Munch et al., 2008; Walther et al., 2010; Erb et al., 2012). The main challenge here is to develop processing techniques that can lead to intricate microstructures resembling those found in natural systems.

In the Studart's group in Switzerland, simple colloid assembling techniques have been developed to control the microstructure of polymer-based composites reinforced with platelets (Erb et al., 2012; Libanori et al., 2012). By controlling the orientation and positioning of reinforcing alumina platelets functionalized with magnetic nanoparticles, investigators have created composites exhibiting unprecedented bio-inspired architectures, using magnetic fields as low as those obtained from magnetic strips on credit cards (Erb et al., 2012). To generate composites with tailored microstructures, magnetic manipulation of the reinforcing platelets is performed, while the surrounding continuous phase is still in the liquid state, either as a polymer solution, monomeric fluid, or molten polymer. The continuous phase can then be consolidated to fix the orientation and positioning of the reinforcing platelets (Fig. 4). By this technique, a new family of high-performance composites reinforced in 3D has been produced, with microstructures incorporating one or more design principles found in biological materials to achieve unusual combinations of mechanical properties. For instance, epoxy bilayer structures exhibiting a structure analogous to that of our teeth revealed a simultaneous increase of $18 \%$ and $62 \%$ in Vickers hardness and flexural modulus in the outer and inner layers, respectively, as compared with their homogeneous counterparts (Erb et al., 2012). Wear resistance is also improved by the aligning of $1 \mathrm{vol} \%$ alumina platelets out-of-plane in a commercial dental resin (Clearfil AP-X, Kuraray, Japan). Orientation of the platelets in the direction normal to the wear ball decreases the maximum wear by $77 \%$ as compared with the inplane reinforcement. This wear volume is also $45 \%$ lower than that observed in the pure commercial resin (Erb et al., 2012). The better performance of the platelets aligned out-of-plane is ascribed to the strong locking state of the platelets within the surrounding polymer matrix, which effectively impedes the penetration of the wear ball into the material.

These results suggest that the possibility of controlling the orientation and positioning of magnetized anisotropic particles in polymeric matrices is a promising technique for the development of new dental-based materials presenting structures organized in the same fashion as natural materials.

\section{ACKNOWLEDGMENTS}

This work has been supported by grants from the National Institutes of Health-NIDCR (R01 DE16849 and ARRA DE16849-04S1), NCRR (S10RR026645 for MicroXCT and
CTSI-SOS Grant Award \# 000166), and a post-doctoral fellowship from Fonds de recherche en santé du Québec (FRSQ). The authors declare no potential conflicts of interest with respect to the authorship and/or publication of this article.

\section{REFERENCES}

Akcora P, Liu H, Kumar SK, Moll J, Li Y, Benicewicz BC, et al. (2009). Anisotropic self-assembly of spherical polymer-grafted nanoparticles. Nat Mater 8:354-359.

Almahdy A, Koller G, Sauro S, Bartsch JW, Sherriff M, Watson TF, et al. (2012). Effects of MMP inhibitors incorporated within dental adhesives. J Dent Res 91:605-611.

Ash BJ, Siegel RW, Schadler LS (2004). Mechanical behavior of alumina/ poly(methyl methacrylate) nanocomposites. Macromol 37:1358-1369.

Atmeh AR, Chong EZ, Richard G, Festy F, Watson TF (2012). Dentincement interfacial interaction: calcium silicates and polyalkenoates. $J$ Dent Res 91:454-459.

Banerjee A, Kellow S, Cook RJ, Watson TF (2010). An in-vitro evaluation of bond strengths of two adhesive bonding agents to residual dentine after caries removal using three excavation techniques. J Dent 38:480-489.

Banerjee A, Pabari H, Paolinelis G, Thompson ID, Watson TF (2011). An in vitro evaluation of selective demineralised enamel removal using bioactive glass air abrasion. Clin Oral Investig 15:895-900.

Barna E, Boomer B, Kursteiner J, Viatal A, Trzebioatowski O, Koch W, et al. (2005). Innovative, scratch proof nanocomposites for clear coatings. Composites Part A: Applied Science and Manufacturing 36:473-480.

Bertassoni LE, Habelitz S, Kinney JH, Marshall SJ, Marshall GW (2009). Biomechanical perspective on the remineralization of dentin. Caries Res 43:70-77.

Bertassoni LE, Habelitz S, Pugach M, Soares PC, Marshall SJ, Marshall GW Jr (2010). Evaluation of surface structural and mechanical changes following remineralization of dentin. Scanning 32:312-319.

Bertassoni LE, Habelitz S, Marshall SJ, Marshall GW (2011). Mechanical recovery of dentin following remineralization in vitro-an indentation study. J Biomech 44:176-181.

Blackman BR, Kinloch AJ, Sanchez FS, Teo WS, Williams JG (2009). The fracture behaviour of structural adhesives under high rates of testing. Eng Fract Mech 76:2868-2889.

Blackman BR, Cur S, Kinloch AJ, Taylor AC (2013). The development of a novel test method to assess the durability of asphalt road-pavement materials. Int $J$ Adhesion \& Adhesives 42:1-10.

Bonderer LJ, Studart AR, Gauckler LJ (2008). Bioinspired design and assembly of platelet reinforced polymer films. Science 319:1069-1073.

Burwell AK, Thula-Mata T, Gower LB, Habelitz S, Kurylo M, Mo SP, et al. (2012). Functional remineralization of dentin lesions using polymerinduced liquid-precursor process. PloS One 7:e38852.

Cramer NB, Stansbury JW, Bowman CN (2011). Recent advances and developments in composite dental restorative materials. $J$ Dent Res 90:402-416

Da Rosa Rodolpho PA, Donassollo TA, Cenci MS, Loguercio AD, Moraes RR, Bronkhorst EM, et al. (2011). 22-year clinical evaluation of the performance of two posterior composites with different filler characteristics. Dent Mater 27:955-963.

De Munck J, Van den Steen PE, Mine A, Van Landuyt KL, Poitevin A, Opdenakker G, et al. (2009). Inhibition of enzymatic degradation of adhesive-dentin interfaces. J Dent Res 88:1101-1106.

Dittanet P, Pearson RA (2012). Effect of silica nanoparticle size on toughening mechanisms of filled epoxy. Polymer 53:1890-1905.

Dittanet P, Pearson RA (2013). Effect of bimodal particle size distributions on the toughening mechanisms in silica nanoparticle filled epoxy resin. Polymer 54:832-845.

Erb RM, Libanori R, Rothfuchs N, Studart AR (2012). Composites reinforced in three dimensions by using low magnetic fields. Science 335:199-204.

Fan Y, Wen ZT, Liao S, Lallier T, Hagan JL, Twomley JT, et al. (2012). Novel amelogenin-releasing hydrogel for remineralization of enamel artificial caries. J Bioact Compat Polym 27:585-603. 
Gao J, Li J, Benicewicz BC, Zhao S, Hillborg H, Schadler LS (2012). The mechanical properties of epoxy composites filled with rubbery copolymer grafted $\mathrm{SiO}_{2}$. Polymers 4:187-210.

Gower LB (2008). Biomimetic model systems for investigating the amorphous precursor pathway and its role in biomineralization. Chem Rev 108:4551-4627.

Irwin PC, Cao Y, Bansal A, Schadler LS (2003). Thermal and mechanical properties of polyimide nanocomposites. Conference on Electrical Insulation and Dielectric Phenomena (CEIDP), pp. 120-123. (DOI:10.1109/CEIDP.2003.1254809).

Jackson AP, Vincent JF, Turner RM (1988). The mechanical design of nacre. Proc R Soc Lond Ser B-Biol Sci 234:415-440.

Johnsen BB, Kinloch AJ, Mohammed RD, Taylor AC (2007). Toughening mechanisms of nanoparticle-modified epoxy polymers. Polymer 48:530-541.

Kawaguchi T, Pearson RA (2003). The effect of particle-matrix adhesion on the mechanical behavior of glass filled epoxies. Part 2. A study on fracture toughness. Polymer 44:4229-4238.

Kinney JH, Pople JA, Driessen CH, Breunig TM, Marshall GW, Marshall SJ (2001). Intrafibrillar mineral may be absent in dentinogenesis imperfecta type II (DI-II). J Dent Res 80:1555-1559.

Kinney JH, Habelitz S, Marshall SJ, Marshall GW (2003). The importance of intrafibrillar mineralization of collagen on the mechanical properties of dentin. $J$ Dent Res 82:957-961.

Lee JS, Yee AF (2000). Role of inherent matrix toughness on fracture of glass bead filled epoxies. Polymer 41:8375-8385.

Lewis J, Smay JE, Stuecker J, Cesarano J 3rd (2006). Direct ink writing of three-dimensional ceramic structures. J Am Ceram Soc 89:3599-3609.

Libanori R, Erb RM, Reiser A, Le Ferrand H, Süess MJ, Spolenak R, et al. (2012). Stretchable heterogeneous composites with extreme mechanical gradients. Nat Commun 3:1265.

Lin A, McIntyre NS, Davidson RD (1992). Studies on the adhesion of glassionomer cements to dentin. J Dent Res 71:1836-1841.

Lin Y, Stansbury JW (2003). Kinetics studies of hybrid structure formation by controlled photopolymerization. Polymer 44:4781-4789.

Liu JC, Howard GD, Lewis SH, Barros MD, Stansbury JW (2012). A study of shrinkage stress reduction and mechanical properties of nanogelmodified resin systems. Eur Polym J 48:1819-1828.

Liu Y, Mai S, Li N, Yiu CK, Mao J, Pashley DH, et al. (2011). Differences between top-down and bottom-up approaches in mineralizing thick, partially-demineralized collagen scaffolds. Acta Biomater 7:17421751.

Lu H, Trujillo-Lemon M, Ge JH, Stansbury JW (2010). Dental resins based on dimer acid dimethacrylates: a route to high conversion with low polymerization shrinkage. Compend Contin Educ Dent 31(Spec No 2):1-4.

Melo MA, Weir MD, Rodrigues LK, Xu HH (2013). Novel calcium phosphate nanocomposite with caries-inhibition in a human in situ model. Dent Mater 29:231-240.

Mertz-Fairhurst EJ, Curtis JW Jr, Ergle JW, Rueggeberg FA, Adair SM (1998). Ultraconservative and cariostatic sealed restorations: results at year 10. J Am Dent Assoc 129:55-66.

Moraese RR, Garcial JW, Barros MD, Lewis SH, Pfeifer CS, Liu JC, et al. (2011). Control of polymerization shrinkage and stress in nanogelmodified monomer and composite materials. Dent Mater 27:509-519.

Munch E, Launey ME, Alsem DH, Saiz E, Tomsia AP, Ritchie RO (2008). Tough, bio-inspired hybrid materials. Science 322:1516-1520.

Nudelman F, Pieterse K, George A, Bomans PH, Friedrich H, Brylka LJ, et al. (2010). The role of collagen in bone apatite formation in the presence of hydroxyapatite nucleation inhibitors. Nat Mater 9:1004-1009.
Paddick JS, Brailsford SR, Kidd EA, Beighton D (2005). Phenotypic and genotypic selection of microbiota surviving under dental restorations. Appl Environ Microbiol 71:2467-2472.

Pearson RA, Bacigalupo LN (2011). Epoxies toughened with self-assembling block copolymers. ACS 241st National Meeting. Anaheim, CA. URL accessed on 7/29/2013 at: http://www.adhesionsociety.org/wp-content/ uploads/2013-Annual-Meeting-Abstracts/Chen_Epoxies_2013.pdf.

Pearson RA, Yee AF (1989). Toughening mechanisms in elastomer-modified epoxies - Part 3: The effect of crosslink density. J Mater Sci 24:2571.

Peters MC, Bresciani E, Barata TJ, Fagundes TC, Navarro RL, Navarro MF, et al. (2010). In vivo dentin remineralization by calcium-phosphate cement. J Dent Res 89:286-291.

Qi YP, Nan L, Niu L, Primus CM, Ling JQ, Pashley DH, et al. (2012). Remineralization of artificial dentinal caries lesions by biomimetically modified mineral trioxide aggregate. Acta Biomater 8:836-842.

Ravichandran R, Ng CH, Liao S, Pliszka D, Raghunath M, Ramakrisnha S, et al. (2012). Biomimetic surface modification of titanium surfaces for early cell capture by advanced electrospinning. Biomed Mater 7:015001.

Rose LR (1987). Toughening due to crack-front interaction with a secondphase dispersion. Mech Mater 6:11-15.

Sides SW, Kim BJ, Kramer EJ, Fredrickson GH (2006). Hybrid particlefield simulations of polymer nanocomposites. Phys Rev Lett 96:250601.

Stansbury JW, Dickens SH (2001). Network formation and compositional drift during photo-initiated copolymerization of dimethacrylate monomers. Polymer 42:6363-6369.

Stefik M, Wang S, Hovden R, Tate MW, Muller DA, Gruner SM, et al. (2012). Networked and chiral nanocomposites from ABC triblock terpolymer coassembly with transition metal oxide nanoparticles. J Mater Chem 22:1078-1087.

Studart AR (2012). Towards high-performance bioinspired composites. $A d v$ Mater 24:5024-5044.

Tanaka J, Hashimoto T, Stansbury JW, Antonucci JM, Suzuki K (2001). Polymer properties of resins composed of UDMA and methacrylates with the carboxyl group. Dent Mater J 20:206-215.

Tang ZY, Kotov NA, Magonov S, Ozturk B (2003). Nanostructured artificial nacre. Nat Mater 2:413-418.

Tay FR, Pashley DH (2008). Guided tissue remineralisation of partially demineralised human dentine. Biomaterials 29:1127-1137.

ten Cate JM (2001). Remineralization of caries lesions extending into dentin. J Dent Res 80:1407-1411.

Tran PA, Sarin L, Hurt RH, Webster TJ (2009). Opportunities for nanotechnology-enabled bioactive bone implants. J Mater Chem 19:2653-2659.

Wada S, Yazawa A, Hoshina T, Kameshima Y, Kakemoto H, Tsurumi T, et al. (2008). Preparation of barium titanate nanoparticle sphere arrays and their dielectric properties. IEEE Trans Ultrason Ferroelectr Freq Control 55:1895-1898.

Walther A, Bjurhager I, Malho JM, Ruokolainen J, Berglund L, Ikkala O (2010). Supramolecular control of stiffness and strength in lightweight high-performance nacre-mimetic paper with fire-shielding properties. Angewandte Chemie-Int Edit 49:6448-6453.

Watson TF, Pilecki P, Cook RJ, Azzopardi A, Paolinelis G, Banerjee A, et al. (2008). Operative dentistry and the abuse of dental hard tissues: confocal microscopical imaging of cutting. Oper Dent 33:215-224.

Weir MD, Chow LC, Xu HH (2012). Remineralization of demineralized enamel via calcium phosphate nanocomposite. J Dent Res 91:979-984.

Xu HH, Weir MD, Sun L, Moreau JL, Takagi S, Chow LC, et al. (2010). Strong nanocomposites with $\mathrm{Ca}, \mathrm{PO}(4)$, and $\mathrm{F}$ release for caries inhibition. $J$ Dent Res 89:19-28. 